cones projecting $C$ and $C^{\prime}$ from $(0,0,1,0)$ have contact of at least order $n+1$. Moreover, by changing this vertex to the point $(a, b, 1,0)$ it is easily shown by a method similar to that used in the general case that the cones projecting $C$ and $C^{\prime}$ from any point in the osculating plane have contact of order $n+1$. In other words, this special case arises when the principal plane coincides with the osculating plane.

The UnIVERSity OF Kansas

\title{
ON RECTIFIABILITY IN METRIC SPACES
}

\author{
BY W. A. WILSON
}

1. Introduction. In Menger's studies in metrical geometry* considerable attention is given to the rectification of the simple arc and various definitions of the length of such an arc are discussed. With the definition of arc-length it is then possible to give conditions for the "Konvexifizierbarkeit" of a compact metric space (p. 96) and for the existence of a geodetic arc in a compact metric space (p. 492). Both theorems involve the assumption of the existence of a rectifiable arc between each pair of points. It is intended in this paper to show that these results and some others are due to space properties which are of a more general nature, at least formally, and which suggest possible further studies.

2. Intrinsic Distance. If $a$ and $b$ are two points of a metric space $Z$, we let $a b$ denote the distance between them. A finite set of points $\left\{a_{i}\right\}$ such that $a_{0}=a, a_{n}=b$, and every $a_{i} a_{i+1}<\delta$ will be called a $\delta$-chain from $a$ to $b$, and $a a_{1}+a_{1} a_{2}+\cdots+a_{n-1} b$ will be called its length. If we set $l_{\delta}(a, b)$ equal to the lower bound of the lengths of all $\delta$-chains from $a$ to $b$, it is clear that this number exists if there is any such chain, that it is greater than or equal to $a b$, and that it increases monotonely as $\delta \rightarrow 0$. The upper bound of $l_{\delta}(a, b)$ for all values of $\delta$ is called the intrinsic distance $\nmid$ from $a$ to $b$ and is denoted by $l(a, b)$.

* Untersuchungen über allgemeine Metrik, Mathematische Annalen, vol. 100, pp. 75-163 and vol. 103, pp. 466-501. See also Annals of Mathematics, vol. 32, pp. 739-746.

$\dagger$ This turns out to be essentially the same thing as Menger's "geodetic distance," loc. cit., p. 492 . See $\$ \S 4$ and 7 below. 
For $l(a, b)$ to exist, finite or infinite, it is necessary and sufficient that $Z$ be $\rho$-connected between $a$ and $b$ for every $\rho>0$. If $l(a, b)$ is always finite, it is clear that the metric axioms are satisfied. In general the infinite case will be excluded and the metric space $Z$ will be called intrinsically metric if $l(a, b)$ exists and is finite for every pair of points. The definition gives at once the following theorem.

TheOREM 1. For a metric space to be intrinsically metric it is necessary and sufficient that for each pair of points $a$ and $b$, some $G>0$, and every $\delta>0$, there is a $\delta$-chain from $a$ to $b$ of length less than $G$.

It should be noted that the value of $G$ depends on $a$ and $b$. Even if the metric space $Z$ is compact, there needs be no upper bound to $G$ as $a$ and $b$ run over $Z$. For example let $Z$ be the union of a segment $a b$ and an enumerable set $\left\{C_{i}\right\}$ of arcs from $a$ to $b$, rectifiable, having only the points $a$ and $b$ in common and converging to $a b$, and so constructed that on each $C_{i}$ there are points $a_{i}$ and $b_{i}$ such that $l\left(a, a_{i}\right), l\left(a_{i}, b_{i}\right)$, and $l\left(b_{i}, b\right)$ all approach infinity with $i$. Then the number $G_{i}$ associated with $a_{i}$ and $b_{i}$ also approaches infinity.

3. TheOREM 2. Let $C$ be a simple arc from $a$ to $b$. Then the intrinsic distance from $a$ to $b$ in $C$ is the length of the arc.

Proof. If $s$ is the length of the arc, it is by the classical definition the upper bound of the lengths of all finite chains ordered from $a$ to $b$. As $l_{\delta}(a, b)$ is the lower bound of the lengths of all $\delta$-chains from $a$ to $b$, we have for every $\delta, l_{\delta}(a, b) \leqq s$; whence $l(a, b) \leqq s$.

For any $\epsilon>0$ there is an ordered chain $\left\{a_{i}\right\}$ of length not less than $G-\epsilon / 2$, where $G=s$ if $s$ is finite, and $G$ is arbitrarily large if $s$ is infinite. These points divide $C$ into $n$ arcs $\left\{C_{i}\right\}$, where $C_{i}=a_{i} a_{i+1}$. It follows from the properties of the simple arc that, for any positive $\sigma<\epsilon / 2$ and for a small enough positive $\delta$, no two consecutive points of any $\delta$-chain $K=\left\{c_{r}\right\}$ from $a$ to $b$ lie on non-consecutive arcs $\left\{C_{i}\right\}$ and, if two consecutive points $c_{r}$ and $c_{r+1}$ lie on consecutive arcs $C_{i-1}$ and $C_{i}, a_{i} c_{r}<\sigma /(2 n)$ and $a_{i} c_{r+1}<\sigma /(2 n)$.

Hence, if $c_{r-1}$ denotes the last point of $K$ in $C_{i-1}$ and $c_{t+1}$ denotes the first point of $K$ following $c_{r-1}$ and lying in $C_{i+1}$, we can easily show that the subchain $c_{r}, c_{r+1}, \cdots, c_{t}$ lies wholly in 
$C_{i}, c_{r} a_{i}<\sigma /(2 n), c_{t} a_{i+1}<\sigma /(2 n)$, and the length of this sub-chain is at least $a_{i} a_{i+1}-\sigma / n$. Consequently the length of $K$ is at least $\Sigma a_{i} a_{i+1}-\sigma \geqq G-\epsilon$. Thus for $\delta$ small enough $l_{\delta}(a, b) \geqq G-\epsilon$ and so $l(a, b) \geqq G-\epsilon$. Hence $l(a, b)=s$ if $s$ is finite and it is infinite if $s$ is infinite.

4. TheORem 3. Let $Z$ be a metric space and let there be a simple arc of finite length between the points $a$ and $b$. Then $l(a, b)$ is finite and does not exceed the length of the given arc.

Proof. Let $C$ be the given arc and let $s$ be its length. For any positive $\delta$ there is an integer $n$ such that $s / n<\delta$. Since $C$ is rectifiable, it can be divided into $n$ arcs each of length $s / n$ by points $\left\{a_{i}\right\}$. These points, together with $a$ and $b$, form a $\delta$-chain of length not more than $s$. It follows at once that $l(a, b) \leqq s$.

COROLlARY. The existence of a rectifiable arc between each pair of points of a metric space is sufficient to make the space intrinsically metric.

5. TheOrem 4. Let $Z$ be a metric $W$-space* and for the points $a$ and $b$ let $l(a, b)$ be finite. Then there is a point $c$ such that $l(a, c)=l(c, b)=l(a, b) / 2$.

Proof. Let $\left\{\epsilon_{i}\right\}$ and $\left\{\delta_{i}\right\}$ denote descending sequences of positive numbers converging to 0 . By the definition of $l(a, b)$ there is for each $i$ a $\delta_{i}$-chain $C_{i}$ from $a$ to $b$, whose length $L\left(C_{i}\right)$ satisfies the relation $\left|l(a, b)-L\left(C_{i}\right)\right|<\epsilon_{i}$. Clearly some point $c_{i}$ of the chain $C_{i}$ breaks it up into two chains $E_{i}$ and $F_{i}$, which join $a$ and $c_{i}$, and $c_{i}$ and $b$, respectively, and satisfy the relations

$$
\left|L\left(E_{i}\right)-L\left(C_{i}\right) / 2\right|<\delta_{i} ;\left|L\left(F_{i}\right)-L\left(C_{i}\right) / 2\right|<\delta_{i} .
$$

Consequently both $L\left(E_{i}\right)$ and $L\left(F_{i}\right)$ differ from $l(a, b) / 2$ by less than $\epsilon_{i}+\delta_{i}$.

As all the points of these chains lie within a finite distance from $a$, there is a sub-sequence for which $\left\{c_{i}\right\}$ converges to a point $c$. Let $\eta_{i}$ be the larger of the two numbers $\delta_{i}$ and $2 c c_{i}$. Then the sum of $E_{i}$ and the point $c$ forms an $\eta_{i}$-chain from $a$ to $c$ of length less than $l(a, b) / 2+\epsilon_{i}+2 \eta_{i}$. But $\eta_{i} \rightarrow 0$ as $i \rightarrow \infty$.

* This is a metric space in which the Bolzano-Weierstrass principle that every bounded infinite set has at least one limiting point is valid. In such a space every bounded closed set is compact. 
Consequently $l(a, c)$ is finite and not larger than $l(a, b) / 2$. In like manner $l(c, b) \leqq l(a, b) / 2$.

Since we know that for any three points $l(a, c)+l(c, b) \geqq l(a, b)$, these last relations give the theorem.

Corollary. A metric W-space which is intrinsically metric is intrinsically convex.

6. Theorem 5. Let $Z$ be a metric $W$-space and for the points $a$ and $b$ let $l(a, b)$ be finite. Then $Z$ contains $a$ simple arc joining $a$ and $b$.

Proof.* By $\S 5$ there is a point $c$ such that $l(a, c)=l(c, b)=$ $l(a, b) / 2$. Again there is a point $d$ such that $l(a, d)=l(d, c)=$ $l(a, c) / 2=l(a, b) / 4$. Now $l(a, d)+l(d, b) \geqq l(a, b)$, whence $l(d, b) \geqq \frac{3}{4} l(a, b)$. But $l(d, b) \leqq l(d, c)+l(c, b)=\frac{3}{4} l(a, b)$. Thus $l(d, b)=\frac{3}{4} l(a, b)$. By mathematical induction we get an enumerable set of points $\{x\}$ such that, if we take $l(a, b)$ as unity and let $t$ vary from 0 to 1 , there corresponds uniformly one point $x$ to each dyadically rational value of $t, l(a, x)=t$, and $l(x, b)=$ $1-t$, while for $t^{\prime}>t$, say, also $l\left(a, x^{\prime}\right)=l(a, x)+l\left(x, x^{\prime}\right)$ and $l(x, b)=l\left(x, x^{\prime}\right)+l\left(x^{\prime}, b\right)$.

Now let $\tau$ be any value of $t,\left\{t_{i}\right\}$ be a sequence of dyadic rationals approaching $\tau$, and $x_{i}$ correspond to $t_{i}$. Since every bounded closed sub-set of $Z$ is compact, we have a sub-sequence of points $\left\{x_{i}\right\}$ converging to a point $c$. Now $l(a, c) \leqq \lim$ $l\left(a, x_{i}\right)=\tau$ and $l(c, b) \leqq \lim l\left(x_{i}, b\right)=1-\tau$. Then $l(a, b) \leqq l(a, c)+$ $l(c, b) \leqq l(a, b)$; whence $l\left(a, x_{i}\right) \rightarrow l(a, c)$ and $l\left(x_{i}, b\right) \rightarrow l(c, b)$. Let $\left\{t_{i}^{\prime}\right\}$ be another sequence of dyadic rationals approaching $\tau$, let $y_{i}$ correspond to $t_{i}^{\prime}$, and $y_{i} \rightarrow c^{\prime}$, where $c^{\prime} \neq c$. Then $\tau=\lim$ $l\left(a, y_{i}\right)=l\left(a, c^{\prime}\right)$ and $1-\tau=\lim l\left(y_{i}, b\right)=l\left(c^{\prime}, b\right)$. For some sub-sequence $l\left(a, x_{i}\right) \leqq l\left(a, y_{i}\right)$ or $l\left(a, y_{i}\right) \leqq l\left(a, x_{i}\right)$, say the former. For these points $l\left(a, y_{i}\right)=l\left(a, x_{i}\right)+l\left(x_{i}, y_{i}\right)$. Now $l(a, c)=$ $l\left(a, c^{\prime}\right)$, which shows that $l\left(x_{i}, y_{i}\right) \rightarrow 0$. Hence $x_{i} y_{i} \rightarrow 0$, which is impossible unless $c=c^{\prime}$. Thus for every sequence $\left\{t_{i}\right\}$ converging to $\tau$, the corresponding sequence $\left\{x_{i}\right\}$ converges to a single point $c$, for which $l(a, c)=\tau$ and $l(c, b)=1-\tau$.

Let $C$ be the union of the sets $\{x\}$ and $\{c\}$. Then $C$ is in ane to one correspondence with the set $0 \leqq t \leqq 1$. Let $x$ now denote

* This proof is much like that given by Menger (loc. cit., p. 88) for the existence of a geodetic arc in a complete convex space. See also pp. 492-493. 
any point of $C$ and $x=f(t)$. Let $\tau$ be any value of $t,\left\{t_{i}\right\}$ be any sequence converging to $\tau$, and $x_{i}=f\left(t_{i}\right)$. By the previous paragraph there is for any $\epsilon>0$ a dyadic rational $t_{i}{ }^{\prime}$ such that $\left|t_{i}-t_{i}^{\prime}\right|<\epsilon / 2^{i}$ and, if $y_{i}=f\left(t_{i}^{\prime}\right), x_{i} y_{i}<\epsilon / 2^{i}$. Then $t_{i}^{\prime} \rightarrow \tau$ and the sequence $\left\{y_{i}\right\}$ converges to $c=f(\tau)$. Since $x_{i} y_{i} \rightarrow 0$, this gives $x_{i} \rightarrow c$. Thus $f(t)$ is continuous.

Since the inverse of $x=f(t)$ is one-valued and the linear interval is compact, the function $f(t)$ defines a homeomorphism and so $C$ is a simple arc.

7. THEOREM 6. Let $Z$ be a metric $W$-space and for the points a and $b$ let $l(a, b)$ be finite. Then there is a rectifiable arc from $a$ to $b$ of length $l(a, b)$.

Proof. By $\S 6$ there is a simple arc $C$ from $a$ to $b$. As seen in the proof of $\$ 6$, there is for each $\delta>0$ a $\delta$-chain from $a$ to $b$ in $C$ of length not more than $l(a, b)$. Hence $l(a, b)$ in $C$ is not more than $l(a, b)$ in $Z$. It is of course not less. Then by $\S 3$ the length of the arc $C$ is $l(a, b)$, which was to be proved.

8. Examples. If a sub-set $M$ of $Z$ is intrinsically metric, we may use the intrinsic distance to define intrinsic separability, compactness, closure, etc. Thus $a$ is an intrinsic limiting point of $M$ if for every $\epsilon>0$ there is a point $x$ of $M$ differing from $a$ for which $l(a, x)<\epsilon$. Note, however, that separability, etc., in the ordinary sense does not imply intrinsic separability, etc.

EXAMPLE I. Let $M$ be the plane set defined by $0<x<1$, $y=x \sin ^{2}(\pi / x)$ and $y=2 ; x=0$ and $x=1,0 \leqq y \leqq 2$. This is closed and so compact. It is intrinsically closed, but not intrinsically compact. If the open segment parallel to the $x$-axis is deleted, it is no longer intrinsically metric.

EXAMPLE II. Let $\left\{a_{i}\right\}$ be a sequence of points converging to a point $a$ and $\left\{a a_{i}\right\}$ be a set of rectifiable arcs, each of length 1 and diameter $1 / i$, and distinct except for the point $a$. Then $Z=U\left[a a_{i}\right]$ is compact and locally connected, but it is not intrinsically compact. (This example is given by Menger, loc. cit., p. 497.)

ExAmple III. Let $C$ be a Cantor set in the segment $0 \leqq x \leqq 1$, and $I$ be the set of complementary intervals. At each point $x$ of $C$ erect a perpendicular $H_{x}$ of length 1 . The union of $I, C$, and the segments $\left\{H_{x}\right\}$ is separable and intrinsically metric, but it is not intrinsically separable since the end of any $H_{x}$ is approached intrinsically only by points on $H_{x}$. 
Although these transformations of space effected by the use of intrinsic distance seem to make this an artificial and futile concept, it should be remembered that, if these were real spaces, to a dweller in any one of them the intrinsic distance, or something like it, would be the real distance rather than that of the higher space containing his world. The various peculiarities illustrated above are due to the absence of a single property to which we now turn.

9. Local Rectifiability. In each of the examples of $\$ 8$ there is a point $a$ for which the relation $a x \rightarrow 0$ does not imply that $l(a, x) \rightarrow 0$. In Example I it is $(0,0)$, in Example II it is $a$, and in Example III it is any point not on $C+I$. To meet this difficulty we introduce the idea of local rectifiability.

Definition. A metric space $Z$ is locally rectifiable at a point a, if, for every $r>0$, there is a $k>0$ such that the relation $a b<k$ $i m p l i e s$ the existence for every $\delta>0$ of some $\delta$-chain from $a$ to $b$ of length less than $r$.

The following theorems are easily proved.

TheOREM 7. A metric space is locally rectifiable at a point a, if, and only if, for every $r>0$, there is a $k>0$ such that the relation $a b<k$ implies that $l(a, b)<r$.

THEOREM 8. Let $Z$ be a metric $W$-space which is locally rectifiable at a point $a$. Then for every $r>0$ there is a $k>0$ such that, if $a x<k$, there is a rectifiable arc from a to $x$ of length less than $r$.

Theorem 9. In a metric $W$-space local rectifiability is a stronger property than local connectivity.

The first of these is an immediate consequence of $\$ 2$. The second follows from the first and $\$ 7$. That local rectifiability is a stronger property than local connectivity is a consequence of Theorem 8, and that it is effectively stronger follows from Example II of $\$ 8$.

10. THeOREM 10. An everywhere locally rectifiable compact metric space is uniformly locally rectifiable.

Proof. Take a positive $r$ less than half the diameter of the space. It is easily seen from Theorem 7 of the previous section that for each point $a$ there is a largest positive $k$ for which the relation $a x<k$ implies that $l(a, x)<r$. Denote this largest $k$ by 
$k(r, a)$. If the theorem is not true, there is, for some $r>0$, a sequence $\left\{a_{i}\right\}$ converging to a point $a$ for which $k\left(r, a_{i}\right) \rightarrow 0$. But there is a $\delta>0$ for which the relation $a x<\delta$ implies that $l(a, x)<r / 2$. If $a a_{i}<\delta / 2$ and $a_{i} x<\delta / 2, a x<\delta$. Hence $l\left(a_{i}, x\right) \leqq$ $l\left(a, a_{i}\right)+l(a, x)<r$. Thus we have a contradiction, and the theorem is valid.

11. THEOREM 11. If $Z$ is a compact connected metric space which is locally rectifiable at each point, it is intrinsically metric.

Proof. For $r>0$ there is, by $\$ 10$, a $k>0$ such that for every $x$, the relation $x y<k$ implies that $l(x, y)<r$. By the Borel theorem a finite set of the regions $\left\{V_{k}(x)\right\} *$ covers $Z$; let these be $V_{1}, V_{2}, \cdots, V_{n}$. This set of regions forms a connected chain, since $Z$ is a connected set. Hence by $\S 9$, Theorem 8 , any two points of $Z$ can be joined by an arc of length not more than $2 n r$. This, with $\S 4$, gives the theorem.

12. Theorem 12. Let $Z$ be intrinsically metric. Let $Z^{\prime}$ have the same points as $Z$, but let the distance in each case be the intrinsic distance. For $Z$ ' to be homeomorphic with $Z$ it is necessary and sufficient that $Z$ be locally rectifiable.

Proof. Let $Z$ be locally rectifiable. Then the relation $a x \rightarrow 0$ implies that $l(a, x) \rightarrow 0$. But $l(a, x) \geqq a x$. Hence the condition is sufficient. It is obviously necessary.

Corollary 1. An intrinsically metric $W$-space $Z$ can be made convex if it is everywhere locally rectifiable.

This is an immediate consequence of Theorem 12 and $\$ 5$.

COROLlaRy 2. If $Z$ is compact, connected, and everywhere locally rectifiable, it can be made convex.

Corollary 3. Let $Z$ be a metric $W$-space in which any two points can be joined by a rectifiable arc and such that for each $\epsilon>0$ and each point a there is $a \delta=\phi(a, \epsilon)>0$ such that, if $a x<\delta$ there is an arc ax of length less than $\epsilon$. Then $Z$ can be made convex.

For $Z$ is intrinsically metric and locally rectifiable at every point. This corollary is a slight generalization of Menger's theorem (loc. cit., p. 96).

\footnotetext{
* This notation means the set of points of $Z$ whose distances from $x$ are less than $k$.
} 
13. Intrinsic Continuity. It is evident that the properties of being intrinsically metric or locally rectifiable are not always preserved under an ordinary homeomorphism. The question naturally arises as to what kind of a homeomorphism does preserve such properties. The following provides a rather obvious answer, which may, however, suggest further avenues of study.

Let $T=\{t\}$ and $Z=\{x\}$ be metric spaces and $x=f(t)$ be one-valued. For a point $a$ in $T$ whose image in $Z$ is $b$ and for each $\epsilon>0$, let there be a $\delta>0$ such that $l(a, t)<\delta$ implies that $l(b, x)<\epsilon$. Then $f(t)$ is called intrinsically continuous at $a$.

THEOREM 13. Let $T=\{t\}$ and $Z=\{x\}$ be homeomorphic metric spaces and $x=f(t)$ define the correspondence. If $T$ is locally rectifiable at $a$ and $f(t)$ is intrinsically continuous at $a$, then $Z$ is locally rectifiable at $b=f(a)$. Conversely, if $T$ is locally rectifiable at $a$ and $Z$ is locally rectifiable at $b=f(a)$, then $f(t)$ is intrinsically continuous at a.

The direct theorem follows from the fact that each of the following statements implies the next: $b x \rightarrow 0 ; a t \rightarrow 0 ; l(a, t) \rightarrow 0$; $l(b, x) \rightarrow 0$. The converse follows from a similar chain $l(a, t) \rightarrow 0$; $a t \rightarrow 0 ; b x \rightarrow 0 ; l(b, x) \rightarrow 0$.

YALE University 